\title{
A virtual screen identified C96 as a novel inhibitor of phosphatidylinositol 3-kinase that displays potent preclinical activity against multiple myeloma in vitro and in vivo
}

\author{
Juan Tang ${ }^{1,2, *}$, Jingyu Zhu ${ }^{1,2, *}$, Yang $\mathbf{Y u}^{3}$, Zubin Zhang ${ }^{1,2}$, Guodong Chen ${ }^{1,2}$, Xiumin \\ Zhou ${ }^{4}$, Chunhua Qiao ${ }^{3}$, Tingjun Hou ${ }^{1,5}$, Xinliang Mao ${ }^{1,2,6}$ \\ ${ }^{1}$ Department of Pharmacology, College of Pharmaceutical Sciences, Soochow University, Suzhou, China \\ ${ }^{2}$ Cyrus Tang Hematology Center, Jiangsu Institute of Hematology, The First Affiliated Hospital, Soochow University, \\ Suzhou, China \\ 3 Department of Medicinal Chemistry, College of Pharmaceutical Sciences, Soochow University, Suzhou, China \\ ${ }^{4}$ Department of Oncology, The First Affiliated Hospital of Soochow University, Suzhou, China \\ ${ }^{5}$ College of Pharmaceutical Sciences, Zhejiang University, Hangzhou, China \\ ${ }^{6}$ Collaborative Innovation Center of Hematology, Suzhou, China \\ * These authors contributed equally to this work.
}

Correspondence to:

Xinliang Mao, e-mail: xinliangmao@suda.edu.cn

Tingjun Hou, e-mail: tingjunhou@zju.edu.cn

Key words: C96; apoptosis; PI3K/AKT signal pathway; multiple myeloma; virtual screen

Received: December 05, $2013 \quad$ Accepted: May 18, $2014 \quad$ Published: May 22, 2014

Abbreviations: 4E-BP1, elF4E-binding protein 1; DMSO, dimethyl sulfoxide; FITC, fluorescein isothiocyanate; GAPDH, glyceraldehyde 3-phosphate dehydrogenase; IGF-1, insulin-like growth factor-1; IGF-1R, insulin-like growth factor-1 receptor; IMDM, Iscove's modified Dulbecco's medium; MM, multiple myeloma; mTOR, mammalian target of Rapamycin; MTT, 3-(4,5-dimethylthiazol-2yl)-2,5-diphenyltetra-sodium bromide; PARP, poly(ADP-ribose) polymerase; PDB, protein data bank; PI, propidium iodide; PI3K, phosphatidylinositol 3-kinase; PTEN, phosphatase and tensin homolog; SDS, sodium dodecyl sulfate; TBS, Tris-buffered saline.

\section{ABSTRACT}

The phosphatidylinositol 3-kinase (PI3K)/AKT signaling pathway is emerging as a promising therapeutic target for multiple myeloma (MM). In the present study, we performed a virtual screen against 800,000 of small molecule compounds by targeting PI3Ky. C96, one of such compounds, inhibited PI3K activated by insulin-like growth factor-1 (IGF-1), but did not suppress IGF-1R activation. The cell-free assay revealed that $\mathrm{C} 96$ preferred to inhibit PI3Ka and $\delta$, but was not active against AKT1, 2, 3 or mTOR. C96 inhibited PI3K activation in a time- and concentration-dependent manner. Consistent with its inhibition on PI3K/AKT, C96 downregulated the activation of mTOR, P70S6K, 4E-BP1, but did not suppress other kinases such as ERK and C-Src. Inhibition of the PI3K/AKT signaling pathway by C96 led to MM cell apoptosis which was demonstrated by Annexin $\mathbf{V}$ staining and activation of the pro-apoptotic signals. Furthermore, $\mathrm{C} 96$ displayed potent anti-myeloma activity in a MM xenograft model in nude mice. Oral administration of $100 \mathrm{mg} / \mathrm{kg}$ bodyweight almost fully suppressed tumor growth within 16 days, but without gross toxicity. Importantly, AKT activation was suppressed in tumor tissues from C96-treated mice, which was consistent with delayed tumor growth. Thus, we identified a novel PI3K inhibitor with a great potential for MM therapy.

\section{INTRODUCTION}

Multiple myeloma (MM) is a malignancy of plasma cells, a type of white blood cells normally responsible for producing antibodies. This disease is characterized by impaired hematopoesis, renal dysfunction, bone destruction, and eventual death [1]. MM is the second most common hematological malignancy in the U.S. and constitutes $1 \%$ of death related to cancers. Currently, chemotherapy is one of the major treatments for MM, including newly 
marketed proteasome inhibitors and immunomodulators, however, MM remains incurable, and the median survival period is $3-5$ years with conventional treatments [2]. All current drugs inherit limited efficacy and/or severe toxicity or adverse effects [3, 4], therefore, developing novel agents is in an urgent need, especially in targeted drug discovery.

Among the "druggable" targets in MM, the phosphoinositide-3-kinase (PI3K)/AKT signal pathway is of particular interest. PI3K/AKT plays a critical role in regulating a myriad of cell signals involved in cell proliferation, migration and survival of MM [5-7]. There are four isoforms in the Class I PI3K superfamily, including $\mathrm{PI} 3 \mathrm{~K} \alpha, \beta, \delta$ and $\gamma$, all of which have been demonstrated to be overactivated in MM cells [8, 9]. PI3K dysregulation is closely associated with overexpressed growth factors, such as insulin-like growth factor-1 (IGF-1), and cytokines, such as interleukin-6 (IL-6) [10, 11]. These extracellular signals bind to specific receptors (e.g. IGF-1R and IL-6R), and then activate PI3K, which catalyzes the production of $\mathrm{PI}(3,4,5) \mathrm{P} 3$, a key second messenger, that further mediates AKT activation and downstream signals in the MM pathophysiology [12]. The PI3K/AKT signal pathway is highly associated with chemoresistance and poor prognosis of MM, thus being developed as a promising target for MM therapy [8].

With the advances of combinatorial chemistry, cancer biology, informatics technology and bioengineering, several important strategies and techniques have been developed, including structural optimization, high throughput screen, and computer-based in silico screen [13]. A virtual screen belongs to the in silico screens, which utilizes highperformance computing to identify possible drug candidates which are most likely to bind to a drug target, typically a protein receptor or an enzyme. Compared with traditional high throughput screens, virtual screens are reliable, costeffective and time-saving [14]. In the present study, we performed a virtual screen against 800,000 small molecule compounds from ChemBridge and Specs Chemicals libraries by using PI3K $\gamma$ as the subject. PI3K $\gamma$ is frequently expressed in MM cells [8, 9], and several inhibitors of PI3K $\gamma$ have been developed in the preclinical stages for MM therapy, such as CAL-101, IPI-145, BEZ235, and PI-103 [15], which established a rationale for the discovery of PI3K inhibitors. More importantly, the molecular interaction of small chemical inhibitors and PI3K $\gamma$ has been clearly elucidated [16, 17]. Therefore, $\mathrm{PI} 3 \mathrm{~K} \gamma$ is a well established target for the discovery of PI3K inhibitors. After several rounds of screens and cell- and mouse-based studies, C96, one of these compounds, was identified as a promising candidate for MM therapy.

\section{RESULTS}

\section{C96 inhibits PI3K activity}

Because C96 was identified from a virtual screen by using PI $3 \mathrm{~K} \gamma$ as the target against 800,000 compounds as shown in Figure 1, we subsequently verified its inhibitory activity against PI3K in MM cells using AKT phosphorylation as a readout. MM cell lines LP1 and OPM2 were starved overnight before being treated with C96 $(0-100 \mu \mathrm{M})$ or S14161 $(100 \mu \mathrm{M}$, a positive control [6]) for a short period ( $2 \mathrm{hrs})$, followed by IGF-1 stimulation for $15 \mathrm{~min}$. Immunoblotting revealed that C96 significantly suppressed AKT phosphorylation in a concentration-dependent manner in the presence of IGF-1 but had no effects on total AKT expression, which was similar to the effects of S14161, the proven PI3K inhibitor [6] (Figure 2A). In LP1 cells, C96 at $25 \mu \mathrm{M}$ and $50 \mu \mathrm{M}$ led to a $50 \%$ and $90 \%$ decrease in AKT phosphorylation, respectively, in the 2-hr treatment. AKT phosphorylation was also markedly decreased by C96 in OPM2 cells which does not express PTEN, a negative modulator of the PI3K signaling pathway (Figure $2 \mathrm{~A}$, right panel). In the timecourse study, AKT activation was suppressed by C96 at $50 \mu \mathrm{M}$ within $0.5 \mathrm{hrs}(30 \mathrm{~min}$ ) (Figure 2B). These studies suggested that C96 inhibited PI3K activity in a time- and concentration-dependent manner.

The PI3K/AKT plays a critical role in regulating a myriad of downstream effectors [18], of which the most prominent ones are mTOR/p70S6K/4E-BP1. Many PI3K inhibitors eventually modulate cell proliferation and survival by disrupting this particular pathway [19]. To examine whether PI3K inhibition led to deregulation of the mTOR signaling pathway, we further measured the changes of mTOR, p70S6K and 4E-BP1 in MM cell lines LP1, OPM2, and JJN3 in the presence of C96. As shown in Figure 2C, C96 downregulated the phosphorylation levels of these proteins in all examined cell lines in a concentration-dependent manner.

\section{C96 does not inhibit phosphorylation of other kinases}

The above studies demonstrated that C96 inhibited the PI3K/AKT signaling pathway, whether it affects activities of other kinases was not known. To elucidate this specificity, we first evaluated its effects on IGF-1R because IGF-1R as a receptor tyrosine kinase activates PI3K in the presence of IGF-1. To exclude the potential effects of C96 on IGF-1 signaling, LP1 and JJN3 cells were starved overnight followed by C96 or S14161 treatment for $2 \mathrm{hrs}$, and IGF-1 stimulation for $15 \mathrm{~min}$. As expected, IGF-1 triggered AKT phosphorylation but it was suppressed by both C96 and the proven PI3K inhibitor S14161 without affecting total AKT expression (Figure $3 \mathrm{~A}$ ). In this treatment, IGF-1R phosphorylation was sharply induced by IGF-1, but it was not affected by C96 (Figure 3A). This result suggested that C96 inhibited PI3K activity independent of IGF-1R signaling. Next, we evaluated other kinases including non-receptor kinase c-Src and a PI3K parallel kinase ERK which transduces external cellular signals. Both LP1 and JJN3 cells were 


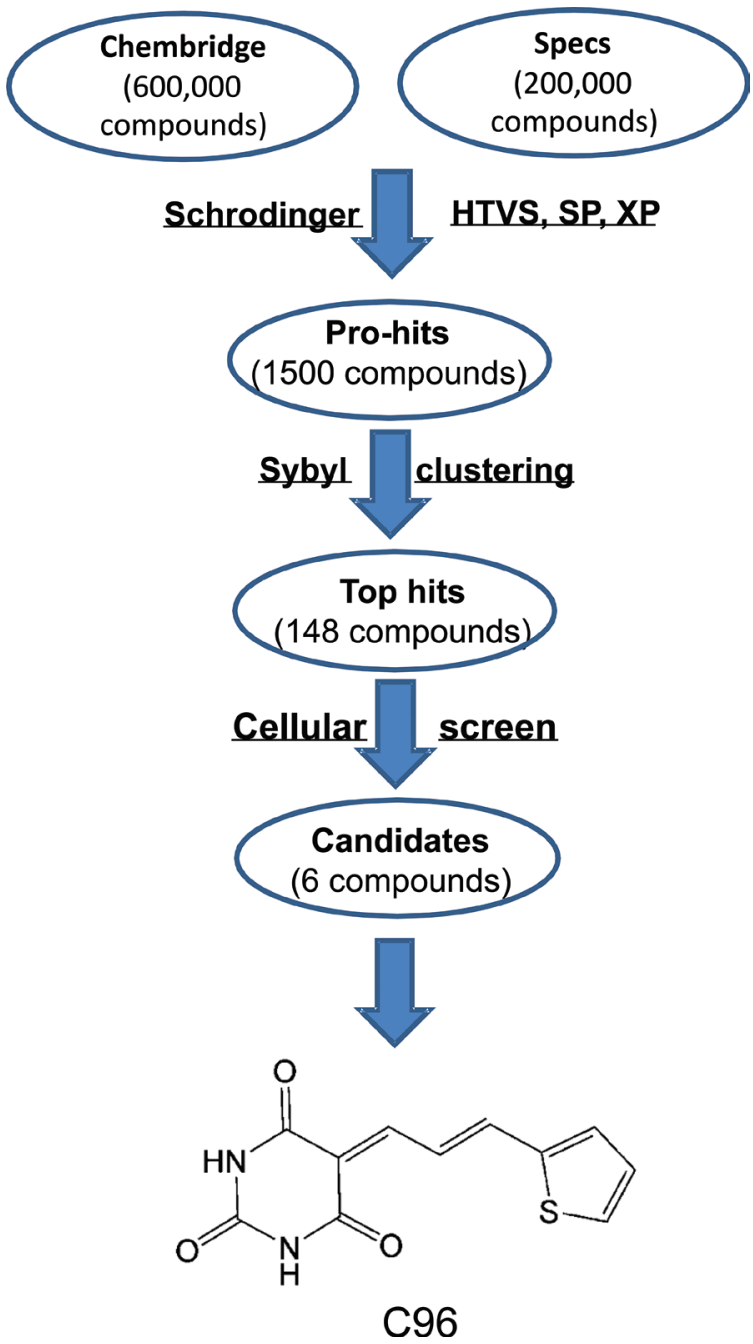

Figure 1: The virtual screening workflow. C96 was generated from a virtual screen using PI3K $\gamma$ as the subject against 800,000 compounds in total from Specs and ChemBridge Chemicals. The molecular docking and scoring were accomplished by using the Schrodinger (Glide), HTVS, SP, and XP modes, followed by Sybyl clustering. Top hits were then verified at the cell-based experiments and singled out for further studies.

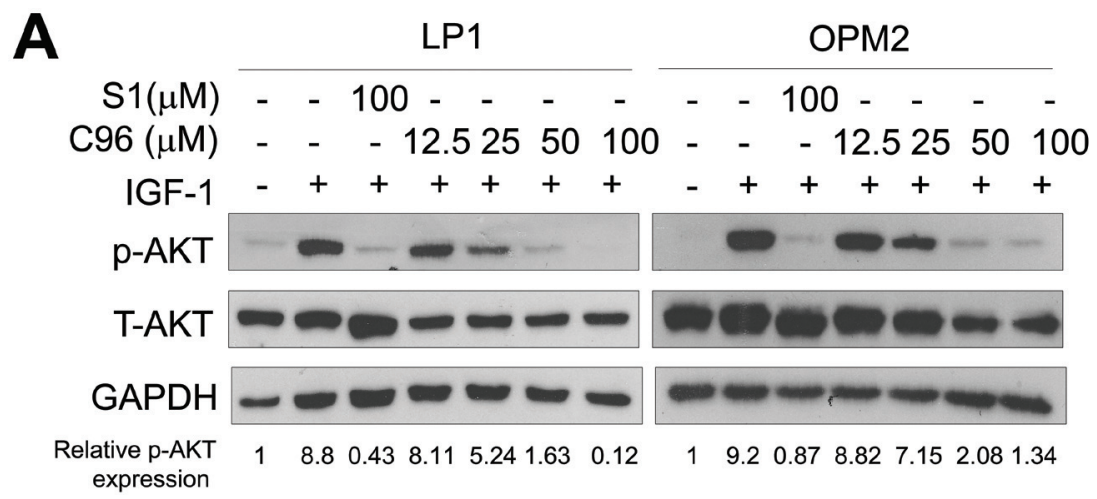

Figure 2: C96 inhibits AKT and mTOR signaling. (A) LP1 and OPM2 were starved overnight, then treated with C96 at the indicated concentrations, or $100 \mu \mathrm{M}$ of S14161 for $2 \mathrm{hrs}$, followed by IGF-1 (100 ng/mL) for $15 \mathrm{~min}$. Cells were collected for the analysis of the expression of p-AKT and total AKT (T-AKT) by immunoblotting.

(Continued) 


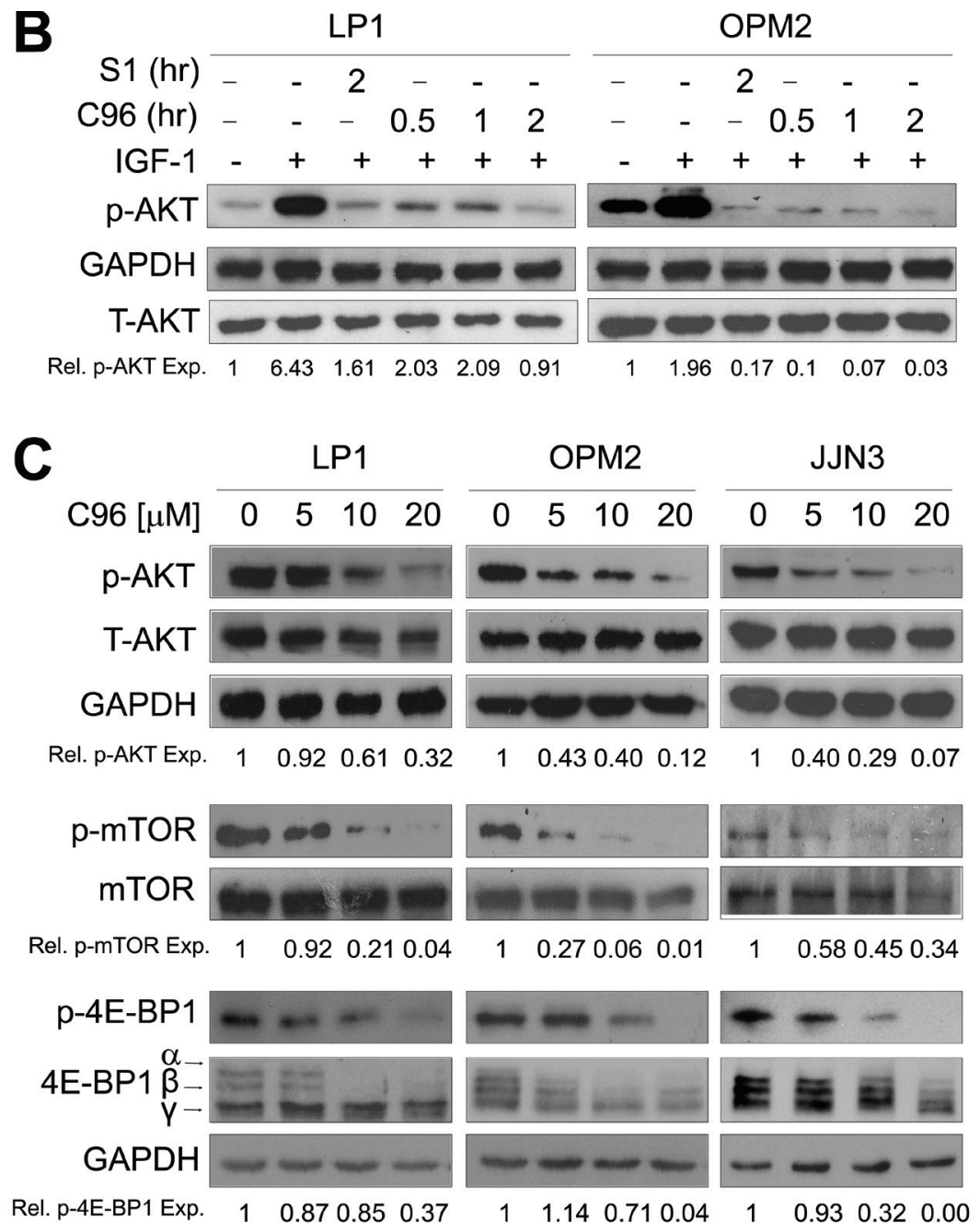

Figure 2: (B) LP1 and OPM2 cells were starved overnight, then treated with C96 (50 $\mu \mathrm{M})$ for different time periods, or S14161 (100 $\mu \mathrm{M})$ for $2 \mathrm{hrs}$, followed by IGF-1 (100 ng/mL) for $15 \mathrm{~min}$. Cells were for the analysis of the expression of p-AKT and T-AKT by immunoblotting. (C) LP1, OPM2, and JJN3 cells were treated with C96 at the indicated concentrations for $24 \mathrm{hrs}$. Cell lysates were prepared and subjected to immunoblotting assay against p-AKT, AKT, p-mTOR, mTOR, p-4E-BP1, and 4E-BP1. GAPDH was used as a loading control.

treated with $\mathrm{C} 96$ at increasing concentrations that inhibited AKT and mTOR activation (Figure 2C). However, C96 did not decrease the phosphorylation level of neither c-Src nor ERK (Figure 3B). These results thus suggested that C96 probably mainly inhibited PI3K/AKT/mTOR signaling pathway.

To confirm this hypothesis, we next evaluated the effects of C96 on PI3K family (PI3K $\alpha, \beta, \delta$, and $\gamma$ ) and associated enzymes including AKT1, 2, 3 and mTOR, in a cell-free enzymatic system as reported previously [6]. It turned out that $\mathrm{C} 96$ preferred to inhibit $\mathrm{PI} 3 \mathrm{~K} \alpha$ and $\delta$ with an $\mathrm{IC}_{50}=5.41$ and $7.05 \mu \mathrm{M}$, respectively, and less activity against $\mathrm{PI} 3 \mathrm{~K} \beta$ and $\gamma\left(\mathrm{IC}_{50}=134\right.$ and $116 \mu \mathrm{M}$, respectively) as shown in Figure 4, but displayed no significant effects on AKT1, 2, 3, or mTOR because all enzymatic activities were higher than $50 \%$ even at $300 \mu \mathrm{M}$ concentration, and their $\mathrm{IC}_{50}$ values were not detectable (Supplemental
Table 1). Taken together, all the above studies suggested C96 was a preferred inhibitor of PI3K, especially the $\alpha$ and $\delta$ isoforms, thus suppressing the PI3K/AKT signaling pathway.

\section{C96 inhibits proliferation and induces apoptosis of MM cells}

Because PI3K/AKT signaling is critical in modulating MM cell proliferation and survival, while interference with this pathway will lead to cell death and regression of MM tumors $[6,8]$, we next asked whether C96 could induce MM cell apoptosis and inhibited MM cell proliferation. MTT assays indicated that C96 suppressed cell proliferation and reduced cell viability of a panel of MM cell lines including LP1, OPM2, JJN3, OCI-My5, RPMI-8226, U266, and KMS11 within 72 hrs 
A
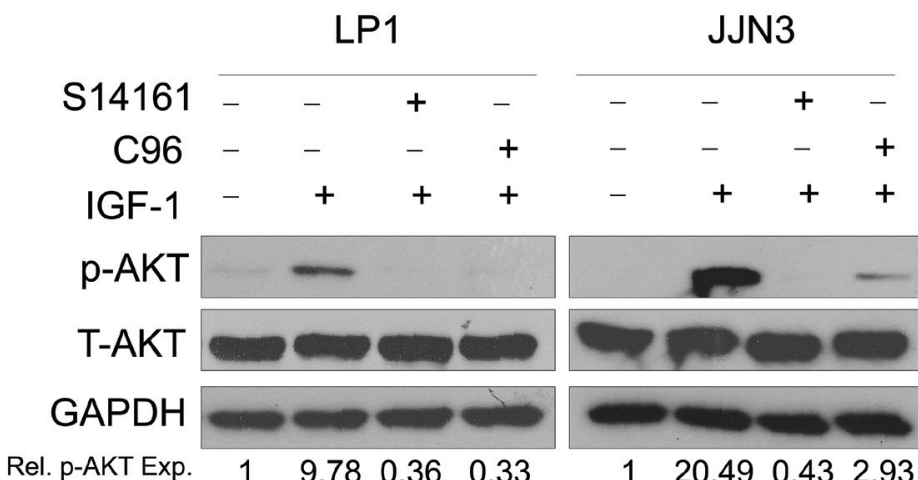

$\begin{array}{llll}1 & 9.78 & 0.36 & 0.33\end{array}$
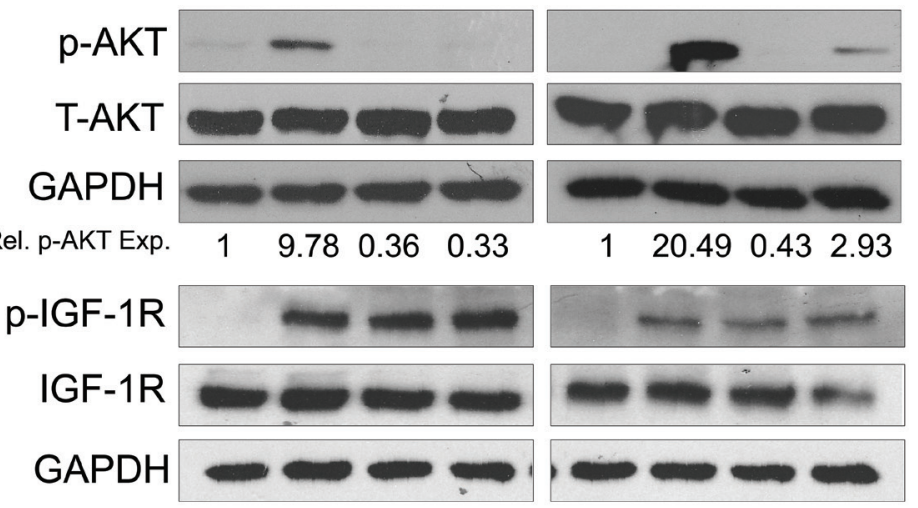

Rel. p-IGF-1R Exp. $\quad 1 \quad 20.8818 .6620 .27$

$1 \quad 2.44 \quad 1.82 \quad 3.25$

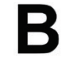

LP1
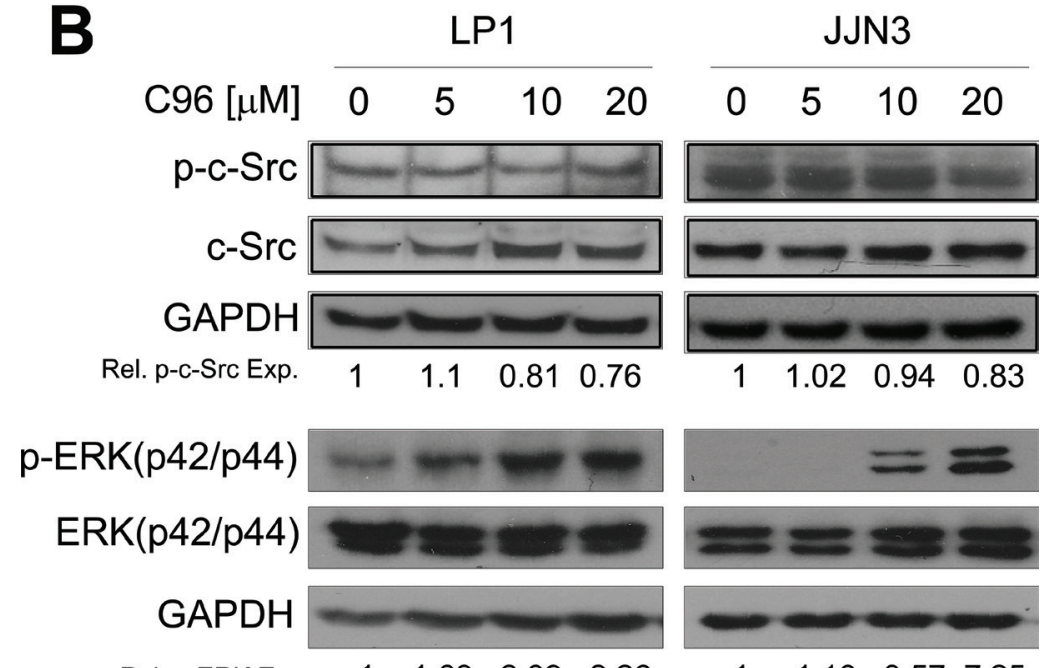

$\begin{array}{lllll}\text { Rel. p-ERK Exp. } \quad 1 & 1.63 & 2.09 & 2.26\end{array}$

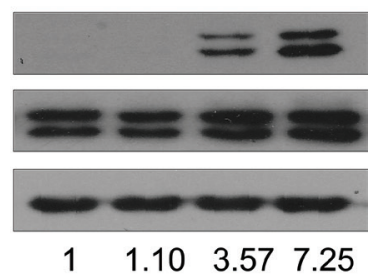

Figure 3: C96 inhibits AKT but not IGF-1R, ERK or c-Src kinase activation. (A) LP1 and JJN3 cells were starved overnight, then treated with $100 \mu \mathrm{M}$ of C96 or S14161 for $2 \mathrm{hrs}$, followed by IGF-1 (100 ng/mL) for $15 \mathrm{~min}$. After incubation, cells were harvested and the expression of p-AKT, p-IGF-1R, total AKT (T-AKT), and IGF-1R proteins were detected by immunoblotting. (B) LP1 and JJN3 were treated with C96 at indicated concentrations for $24 \mathrm{hrs}$ followed by immunoblotting assay for the expression of p-Src, c-Src, p-ERK, and ERK. GAPDH was used as a loading control.

(Supplemental Figure 1). Next, we measured the induction of apoptosis by C96 in MM cell lines. Apoptotic cells are featured with inside-out expression of phosphatidylserine which is specifically identified by Annexin V, a cellular protein in the annexin group [20]. Therefore, to measure the apoptotic level, C96-treated cells were stained with Annexin V-FITC and propidium iodide followed by flow cytometry analysis. As shown in Figure 5, 20 to $60 \%$ of cells underwent apoptosis in the treatment of C96 at $10 \mu \mathrm{M}$ within $24 \mathrm{hrs}$. This result was confirmed by the cleavage of PARP, a biomarker of cell apoptosis (Figure 6A). For example, less apoptotic cells were induced by $\mathrm{C} 96$ in
KMS11 cells in the Annexin V staining assay (Figure 5), the cleavage level of PARP in this cell line was also lower than that seen in other cell lines, such as OPM2 (Figure 6A). We next examined the apoptotic signaling pathway in the treatment with C96 with increasing exposure periods or concentrations. Three cell lines LP1, OPM2, and JJN3 were treated with C96 at $0,5,10$, or $20 \mu \mathrm{M}$ for $24 \mathrm{hrs}$ or $10 \mu \mathrm{M}$ for 3, 6, 9 and $12 \mathrm{hrs}$. Immunoblotting analysis revealed that C96 induced cleavage of PARP and caspase-3 in a time- and concentration-dependent manner (Figure 6C). All the above results demonstrated that C96 induced MM cell apoptosis. 


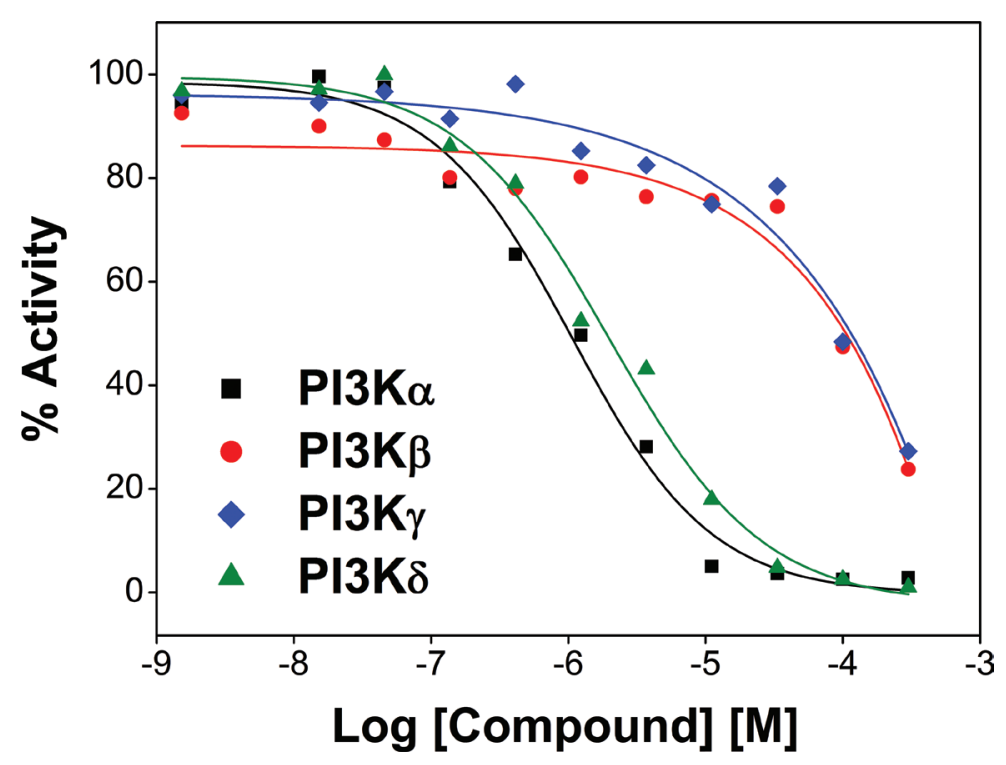

Figure 4: C96 inhibits PI3K activity. PI3K activity analyses in an in vitro cell-free system. Increasing concentrations of S14161 were incubated with the PI3K isoforms $\alpha, \beta, \delta$, and $\gamma$, respectively. Activity of each kinase was determined with HotSpot technology as described in "Kinase activity in cell-free assay".
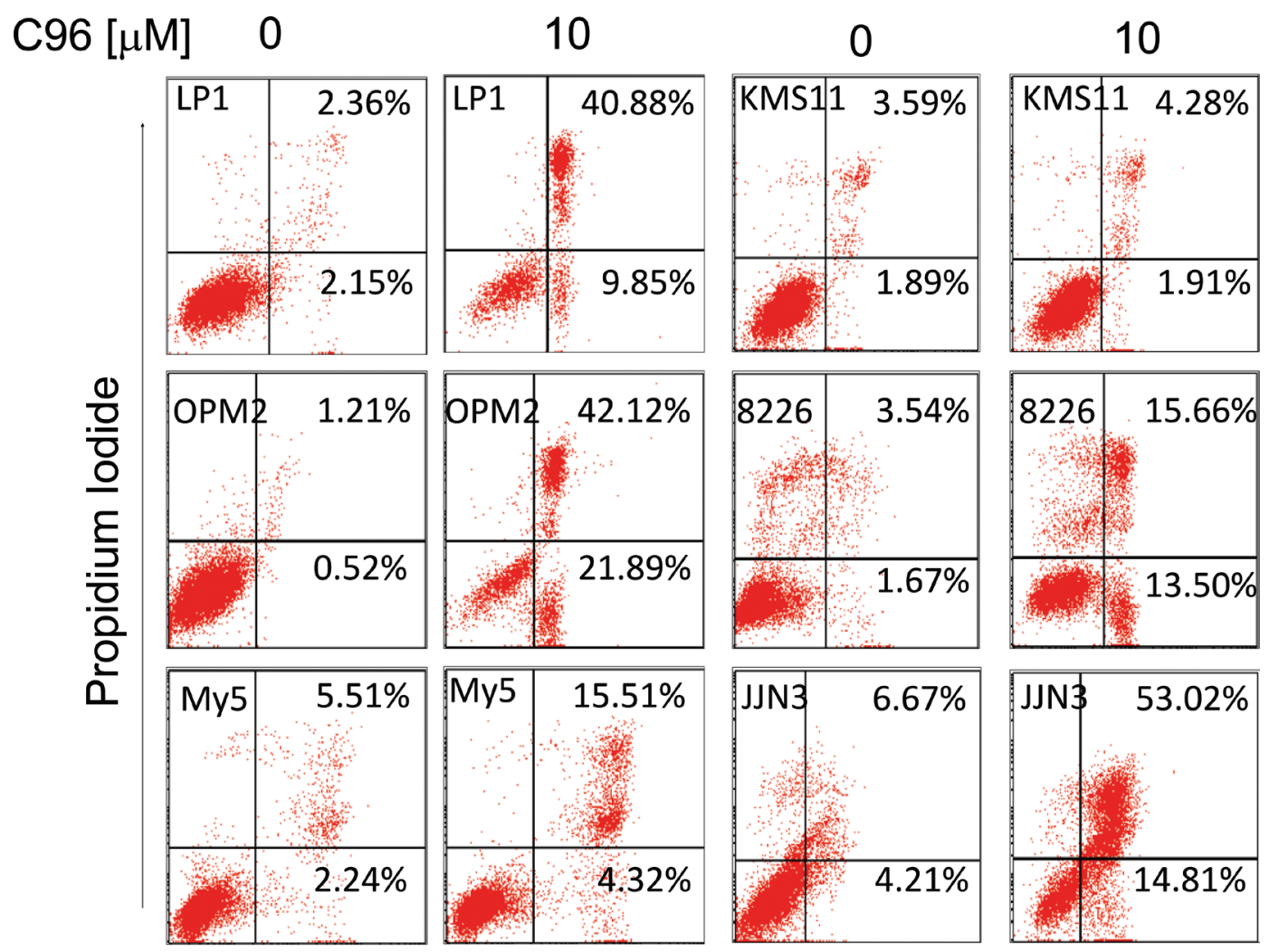

\section{Annexin V-FITC}

Figure 5: C96 induces MM cell apoptosis. MM cell lines LP1, OPM2, OCI-My5 (My5), KMS11, RPMI-8226 (8226), and JJN3 were treated with C96 at 0 or $10 \mu \mathrm{M}$ for $24 \mathrm{hrs}$, followed by propidium iodide and Annexin V-FITC staining and flow cytometric analysis. 


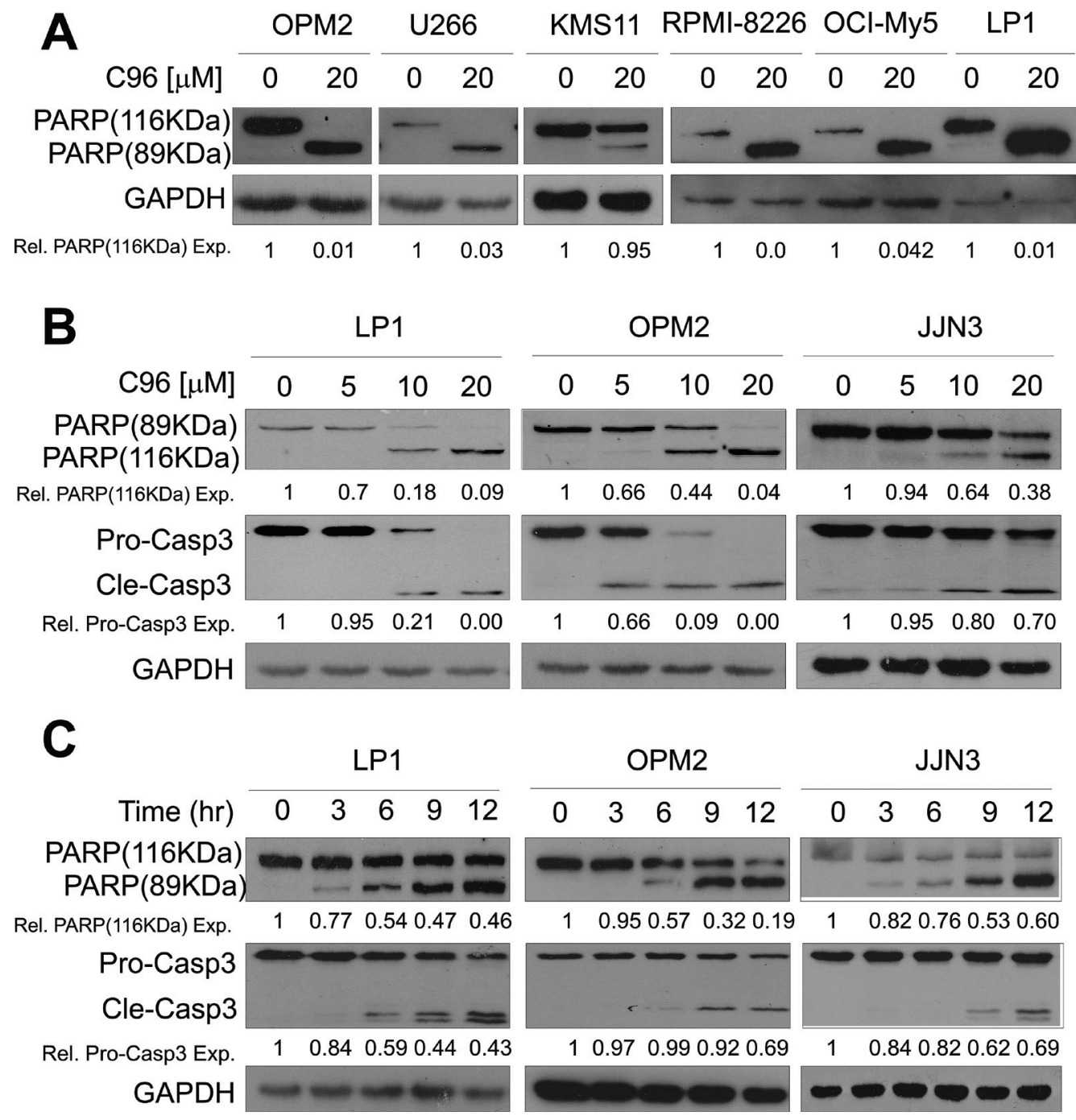

Figure 6: C96 activates apoptotic signaling in MM cells. (A) OPM2, U266, KMS11, RPMI-8226, OCI-My5, and LP1 cells were treated with C96 $(20 \mu \mathrm{M})$ for 24 hrs. Cell lysates were then prepared and subjected to immunoblotting assay against apoptosis-associated proteins PARP, and apoptotic executive enzyme pro-caspase-3 (Pro-Casp3). GAPDH was used as a loading control. (B) LP1, OPM2, and JJN3 were treated with C96 at the indicated concentrations for $12 \mathrm{hrs,} \mathrm{followed} \mathrm{by} \mathrm{immunoblotting} \mathrm{assay} \mathrm{for} \mathrm{pro-caspase-3} \mathrm{(Pro-Casp3),}$ cleaved caspase-3 (Cle-Casp3), and PARP. GAPDH was used as a loading control. (C) LP1, OPM2, and JJN3 were treated with C96 at $10 \mu \mathrm{M}$ for different time points, followed by immunoblotting assay for caspase-3 and PARP. GAPDH was used as a loading control.

Next, we evaluated the effects of C96 on MM cell growth by measuring viable cells after starvation/ IGF-1 stimulation in the presence of C96. As shown in Supplemental Figure 2A, IGF-1 stimulated MM cell growth, but it was suppressed by $\mathrm{C} 96$. We also examined the effects of C96 on MM cell migration upon IGF-1 stimulation. In all three cell lines (LP1, OPM2, and JJN3), LP1 and JJN3 failed to but OPM2 cells could migrate through the 8-micrometer transwell membrane in the presence of IGF-1, and this migration was partly suppressed by C96 (Supplemental Figure 2B). These data suggested that C96 inhibited MM cell mobility in the presence of IGF-1 but it was probably determined by individual cell lines because not all MM cells could migrate through the membrane.

\section{C96 delays tumor growth in a MM xenograft model in association with the inhibition of the PI3K signaling pathway}

To further evaluate the anti-myeloma efficacy of C96 in vivo, a human multiple myeloma xenograft model was established by subcutaneous injection of JJN3 into nude mice. When these tumors were palpable, mice were randomly divided into two groups $(\mathrm{n}=5)$ and one of them were orally administrated C96 (100 mg/kg body weight, 
$1 / 10$ of the oral $\left.\mathrm{LD}_{50}\right)$, the other one were given the same volume of vehicle on a daily base for continued 16 days. Tumor sizes and body weight were monitored every other day. As shown in Figure 7A, C96 delayed MM tumor growth during the experimental period. In contrast, the tumors in the vehicle group grew in a steady rate. However, the 16-day treatment of C96 had minimal effects on body weight (Figure 7B), and there were no gross toxicity observed in all mice treated with C96 (data not shown).

We also measured the changes of the phosphorylation status of AKT and mTOR, two key proteins in the PI3K signaling pathway, in the myeloma tumor tissues. Immunoblotting indicated that both AKT and mTOR phosphorylation was decreased in the myeloma tumor tissues excised from C96-treated mice (Figure 7C), which were consistent with MM tumor growth treated by C96. Therefore, C96 delayed tumor growth of myelomas in vivo, which was associated with the inhibition of the PI3K signaling pathway and was consistent with the in vitro studies.

\section{DISCUSSION}

In the present study, we identified $\mathrm{C} 96$ as a potential PI3K inhibitor via virtual screening and subsequent cellular and mice studies against MM.

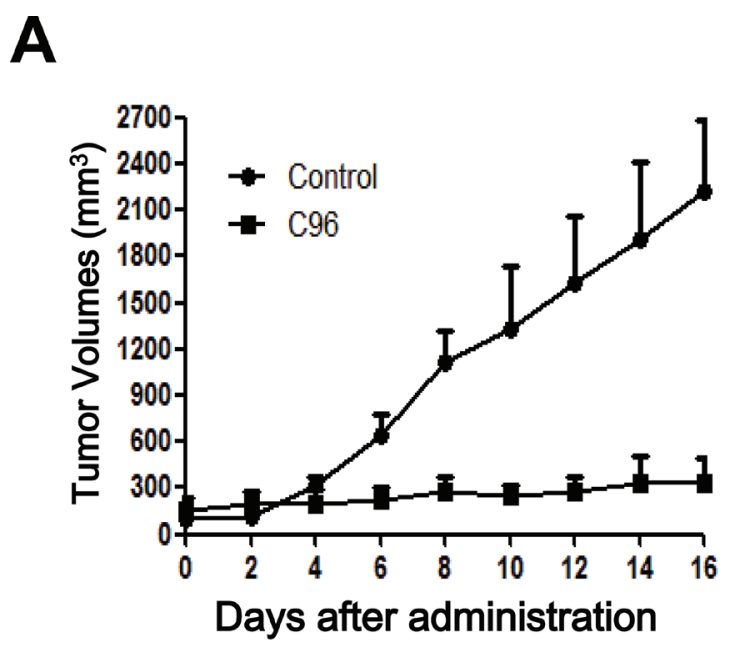

B
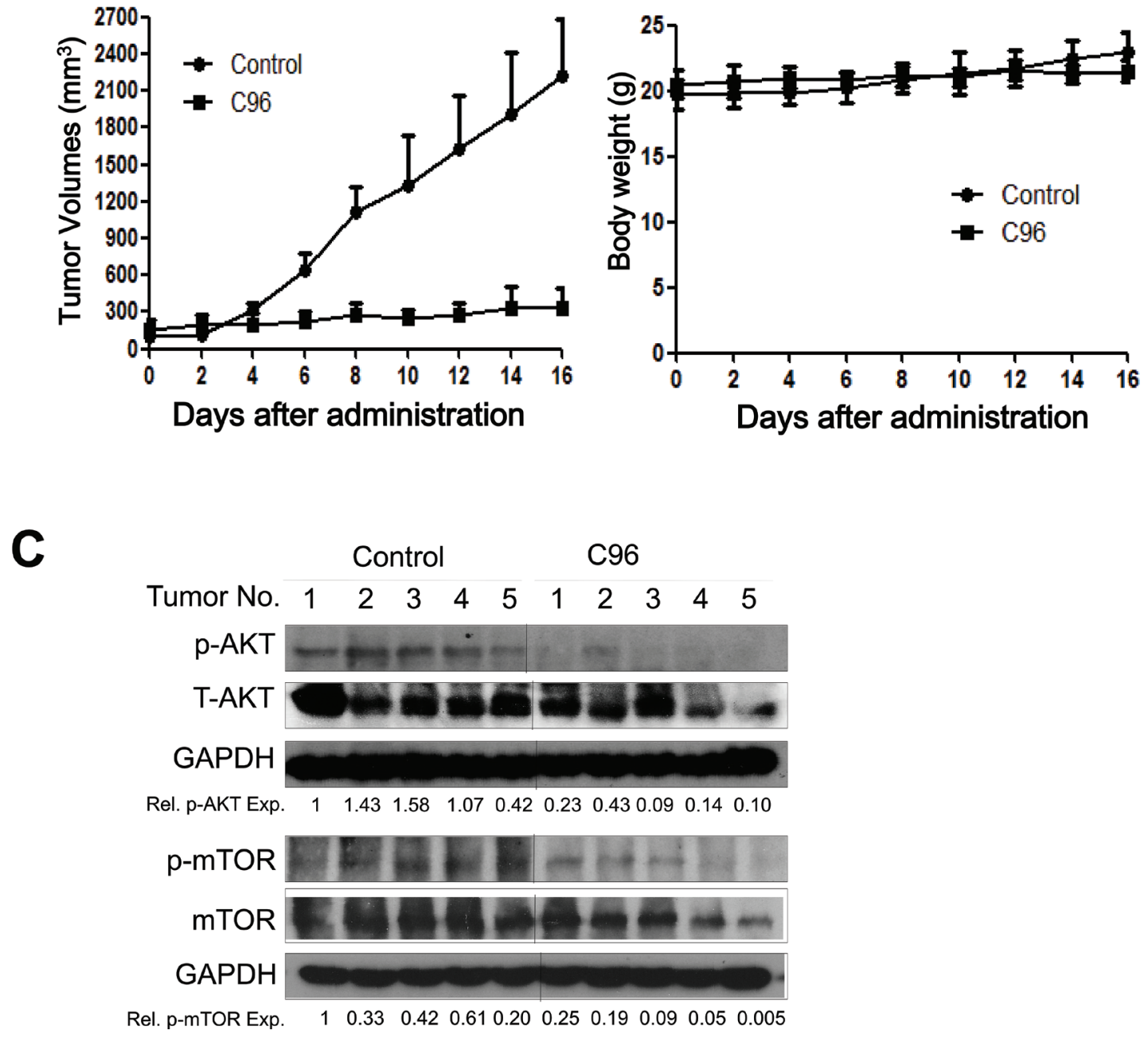

Figure 7: C96 delays myeloma tumor growth in a xenograft mouse model. (A) JJN3 cells were injected subcutaneously into nude mice with a density of 30 million cells/site/mouse. When tumors were palpable, mice (n=5/group) were orally given C96 (100 mg/kg body weight) in PBS containing 10\% Tween 80 and 10\% DMSO daily for continuous 16 days. Tumor volumes were monitored every other day. (B) Mouse body weight was monitored every other day. (C) At the end of the experiment, tumor samples from each group were subjected to immunoblotting analysis for the expression levels of p-AKT, T-AKT, p-mTOR and mTOR with specific antibodies. GAPDH was used as a loading control. 
The PI3K-centered signaling pathway has significance in MM pathophysiology, chemotherapy, as well as clinical prognosis. Consistent with these findings, blockade of the PI3K/AKT signaling pathway with chemical or genetic methods leads to MM cell apoptosis and MM tumor regression [21]. Therefore, targeting PI3K is a key strategy for MM therapy [22]. In the last two decades, robot-assisted large scale targeted drug screens have been significantly implemented due to the advancements in bioinformatics, informative technologies, molecular cancer biology, and synthetic chemistry[13, 23, 24]. In terms of PI3K, the elucidation of crystal structures of $\mathrm{PI} 3 \mathrm{~K}$ isoforms including co-crystallized ligands and apo-structures deposited in the Protein Data Bank (PDB) has made virtual screening possible $[14,25]$. As an attractive possible filter, a virtual screen offers an opportunity to minimize the wet laboratory workload [26]. Using these structure-based virtual screening and extensive docking studies, several PI3K inhibitors have been identified [14, 26, 27]. In the present study, we performed a virtual screen against PI3K $\gamma$ because this isoform has been most studied and its crystal structure has been well defined [16, 28]. Using $\mathrm{PI} 3 \mathrm{~K} \gamma$ as the screening subject against the ChemBridge and Specs chemical libraries, we identified C96 as a potential candidate that was further verified in both cellular and mice models. Consistent with the prediction, C96 inhibits PI3K activation and blocks the PI3K/AKT signaling pathway thus inducing MM cell apoptosis. However, the enzymatic assay in a cell-free PI3K system showed that $\mathrm{C} 96$ prefers to inhibit PI3K $\alpha$ and $\delta$ although it was screened out against the $\gamma$ isoform. This is very interesting but it is highly possible. Firstly, all isoforms share a similar structure of the ATP binding pocket, therefore, there are many important pan-Class I PI3K inhibitors [29]. Secondly, the virtual screen is based on the theoretical interactions between compounds and specific enzymes, however, the actual interactions may vary in the cell-free and in the cell-based assays [30]. After all, the real interaction is determined by the specific physical and chemical properties of the compounds and the specific structure of the receptor enzymes [30]. In the case of C96, it is probable that C96 fits better in the $\alpha$ and $\delta$ active pockets in the native status, such as in cells or in the purified enzymes. We also believe a preferred inhibitor of the $\alpha$ and $\delta$ PI3K isoforms merits further investigation because these two isoforms are overexpressed in MM cells but less expressed in normal blood cells [8] More importantly, the $\gamma$ isoform is required in $\mathrm{T}$ cell functions and in the migration of leukocytes, in particular neutrophils and macrophages [31]. Therefore, the preference of $\mathrm{C} 96$ on the $\alpha$ and $\delta$ isoforms will be more important for the potential application for the treatment of MM. This finding also indicated that the virtual screens must be coupled with wet laboratory investigations, especially at the cellular and at the animal levels. After all, the virtual screen is a theoretical prediction, and the cellular and animal studies must be performed because the "real" human being system is very complicated.

In MM cells, the PI3K signaling is particularly important because it is modulated by various extracellular stimulators, such as the growth factor IGF-1, and the cytokine IL-6, both of which are frequently over-expressed and secreted in MM cells thus stimulating PI3K activation $[10,11]$. IGF-1R activation leads to sustained activation of PI3K thus increasing cell proliferation and survival [32]. Therefore, PI3K inactivation could be due to the effects on the suppression of these membrane signals, such as IGF-1R and IL-6R activation [33]. Some PI3K signaling transduction can be blocked by inactivating IGF-1R and/or IL-6R. However, C96 displays no inhibitory effect on IGF$1 \mathrm{R}$ phosphorylation but markedly inhibits AKT activation, suggesting that C96-mediated PI3K inhibition is not due to its effects on the IGF-1R signaling. More importantly, C96 downregulates PI3K and its subsequent signaling transduction, including the AKT/mTOR pathway, however, its shows no inhibitory effects on other kinases such as c-Src and ERK, suggesting that C96 prefers to inhibit the PI3K/AKT signaling transduction. This is consistent with the cell-free assay in which C96 inhibits the catalytic activity of PI3K but it does not inhibit kinases such as AKT1, 2, 3 or mTOR associated with PI3K. Although C96 has not inhibition on the catalytic activity of AKT or mTOR, it suppressed the phosphorylation of AKT, mTOR, p70S6K, and 4E-BP1, which is probably attributed to PI3K inhibition. Downregulated PI3K/AKT signal transduction leads to suppressed downstream signals, such as the phosphorylation of mTOR and p70S6K, which has been reported previously $[5,34]$. In tumor tissues from a mouse xenograft model established with human MM cell line JJN3, both AKT and mTOR activation was also decreased by C96, which is consistent with studies at the the cellular level, therefore, C96-delayed myeloma tumor growth is consistent with its inhibition on PI3K. These results thus collectively concluded that C96 is a preferred inhibitor of PI3K.

In the last decade, a lot of PI3K inhibitors have been developed and some have shown great potencies in preclinical studies against varied cancer types [18, 29], of which some belong to thiazolidinones, 2-aminothiazoles, or 2-morpholinothiazoles. By comparing with those already known PI3K inhibitors, C96 is featured with a novel chemical structure, therefore it probably represents a novel class of PI3K inhibitors. Moreover, C96 displays a very potent activity in vivo but without marked toxicity. The $\mathrm{EC}_{50} \mathrm{~s}$ against PI3K alpha and delta in the cell-free enzymatic system are around $5 \mu \mathrm{M}$, which is acceptable. More importantly, C96 showed great potency in killing MM cells at low micromolar concentrations and suppresses the growth of MM xenograft in nude mice at a dose less than $1 / 10 \mathrm{LD}_{50}$. At this dose, C96 did not show marked toxicity to mice but suppressed tumor growth 
more than $90 \%$ within 16 days. This is very essential because some known PI3K inhibitors are able to suppress PI3K activity at low nanomolar levels, but they need to reach a concentration up to $10 \mu \mathrm{M}$ to induce MM cell death. For example, BAY80-6946 displays a high activity against all $\mathrm{PI} 3 \mathrm{~K}$ isoforms with $\mathrm{IC}_{50} \mathrm{~s}$ from 0.5 to $6.4 \mathrm{nM}$ in the cell-free enzymatic system, it requires more than 1000 $\mathrm{nM}$ to inhibit MM cell proliferation [9]. Another case is CAL-101, one of the most potent inhibitor of PI3K $\gamma$ and $\delta$, whose $\mathrm{IC}_{50} \mathrm{~s}$ in inhibiting PI3K activity are around $2.5 \mathrm{nM}$, however, it needs more than $10 \mu \mathrm{M}$ to induce $\mathrm{MM}$ cell death [8]. These data indicated that cell-free potency is not consistent with its biological activity, and C96 might have better metabolic parameters than some known PI3K inhibitors.

In a summary, by a virtual screen and further studies at both cellular and mice models, C96 was identified as a novel potent PI3K inhibitor, especially against PI3K $\alpha$ and $\delta$. Because of its efficacy in both in vitro and in vivo and undetectable toxicity in vivo, C96 can be developed as a promising PI3K inhibitor for the treatment of MM, but clinical relevance should be further investigated.

\section{MATERIALS AND METHODS}

\section{Cell culture}

MM cell lines OPM2, RPMI-8226, U266, and KMS11 were obtained from the American Type Culture Collection (Rockville, MD, USA). OCI-My5, LP1, and JJN3 were kindly provided by Dr. Aaron Schimmer from Ontario Cancer Institute, Toronto, Canada. All cell lines were maintained in Iscove's modified Dulbecco's medium (IMDM, Hyclone) supplemented with 10\% fetal bovine serum (Invitrogen, CA), penicillin (100 units $/ \mathrm{mL}$ ) and streptomycin $(100 \mu \mathrm{g} / \mathrm{mL})$, and were grown at $37^{\circ} \mathrm{C}$ in an incubator supplied with $5 \% \mathrm{CO}_{2}$.

\section{Chemicals}

C96 was purchased from ChemBridge Corporation, San Diego, CA. IGF-1 was purchased from PeproTech [5]. Annexin V-FITC, propidium iodide, MTT or 3-(4,5-dimethylthiazol-2-yl)-2,5-diphenyltetra-sodium bromide were purchased from Sigma (St Louis, MO).

\section{Virtual screening}

The crystal structure of PI3K $\gamma$ (PDB entry: 3APC) [35] from the RCSB Brookhaven Protein Data Bank was used as the initial structure for the virtual screening. The databases of 200,000 compounds from Specs (Delft, The Netherlands, http:/www.specs.net/) and 600,000 compounds from ChemBridge (San Diego, United States, http://www.chembridge.com) were used as the initial sources for the screen against PI3K $\gamma$ [14]. The molecular docking calculations were accomplished by using the Glide module in Schrödinger (version 9.0). According to the binding fitness, each compound was assigned a score. Compounds with the highest scores were moved to the next screen with a stricter condition. All structures were docked and scored using the Glide HTVS, SP, XP modes step by step and 1,500 compounds were returned as prohits. Then, chemical similarity clustering of the pro-hits was performed to maximize the chemical diversity of the selected compounds for biological assay using the Selector Module in SYBYL 8.1 and 148 compounds as top-hits were obtained. Lastly, all these 148 compounds were purchased from ChemBridge or Specs, and were applied for cell-based and mouse-based evaluation. C96, 5-(3-(2-thienyl)-2-propen-1-ylidene)-2,4,6(1H,3H,5H)pyrimidinetrione, was chosen for further investigation. The detailed flow chart for the virtual screening was shown in Figure 1.

\section{Growth inhibition assay}

MM cells were dispensed in 96-well plates at a density of $8 \times 10^{3}$ cells per well and treated with increasing concentrations of C96 for $72 \mathrm{hrs}$, and the growth inhibitory effect of C96 on MM cells was assessed by an MTT assay as reported previously [6]. To show the effect of C96 on cell growth in the presence of growth factor IGF-1, MM cells were first maintained in IMDM media containing $0.5 \% \mathrm{FBS}$ for $12 \mathrm{hrs}$, followed by C96 treatment alone or in combination with $100 \mathrm{ng} / \mathrm{mL}$ of IGF-1. Cells were then incubated for another $24 \mathrm{hrs}$, relative cell proliferation was analyzed by an MTT assay.

\section{Flow cytometric analysis of apoptosis}

MM cells treaded with C96 at concentrations from 0 to $20 \mu \mathrm{M}, 24 \mathrm{hrs}$ later, cells were stained with Annexin V-FITC and PI according to the manufacturer's instruction (Sigma, St Louis, MO). Apoptotic cells were measured on a flow cytometer (FACSCalibur, Becton Dickinson) as reported as previously [36].

\section{Kinase activity in cell-free assays}

Kinase activity in the presence of C96 was performed by using the HotSpot technology (Reaction Biology Corp., Malvern, PA, USA) [6]. Briefly, kinases (PI3K $\alpha, \beta, \delta, \gamma, \mathrm{AKT} 1,2,3$, and mTOR) and substrates were diluted in reaction buffer [6]. Subsequently, $5 \mathrm{~nL}$ of serially diluted C96 (in pure DMSO) was delivered into diluted kinase and substrate mixture by using Echo 550 (LabCyte Inc. Sunnyvale, CA). The reaction was started by adding ${ }^{33} \mathrm{P}$-ATP (hot ATP) into the reaction mixture (the final concentration was $10 \mu \mathrm{M}$ ) and stopped after $2 \mathrm{hrs}$ incubation at room temperature. The un-reacted hot ATP was washed away before detection. 


\section{Immunoblotting analysis}

Cells were treated with or without C96 for $24 \mathrm{hrs}$ before being applied to lysate preparation in a lysis buffer $(50 \mathrm{mM}$ Tris-HCI, pH 7.4, $1 \%$ NP-40, $0.5 \%$ Na-deoxycholate, and $0.1 \%$ SDS, $150 \mathrm{mM} \mathrm{NaCl}, 2 \mathrm{mM}$ EDTA, $2 \mathrm{mM} \mathrm{Na}_{3} \mathrm{VO}_{4}$, and $5 \mathrm{mM} \mathrm{NaF}$ ) [6]. Equal amounts $(30 \mu \mathrm{g})$ of total proteins were subjected to sodium dodecyl sulfate-polyacrylamide gel electrophoresis (SDS-PAGE) separation, followed by immunoblotting analyses with specific antibodies. Antibodies against PARP, caspase-3, AKT, p-AKT(S473), 4E-BP1, p-4E-BP1(S65), IGF1R, p-IGF-1R, ERK(p42/p44), and p-ERK(p42/p44) were purchased from Cell Signaling Technologies. Antibodies against mTOR, p-mTOR(S2448), p70S6K, p-p70S6K(S424), and GAPDH were obtained from Abgent (Suzhou, China). Horseradish peroxidase-conjugate secondary antibodies against mouse or rabbit and GAPDH were purchased from Abgent. All immunoblotting signals were further analyzed with Quality One Quality One software (Bio-Rad, Berkeley, CA, USA).

\section{Cell migration analysis by the transwell assay}

This assay was performed as described previously [37]. Briefly, MM cells OPM2, JJN3 and LP1 were serum starved overnight and then re-suspended in $70 \mu \mathrm{L}$ of IMDM medium $/ 0.5 \%$ fetal calf serum with or without C96. These cells were then loaded onto polycarbonate membranes $(8-\mu \mathrm{m}$ pore size) separating 2 chambers of a transwell (Corning Costar, Cambridge, MA). Medium $/ 0.1 \%$ FCS $(500 \mu \mathrm{L})$ containing agonist IGF-1 was added to the lower chamber of the Transwell cluster plates. After 6 hrs, cells migrating into the lower chamber were counted using a hemacytometer.

\section{Myeloma xenograft study}

JJN3 cells (30 million cells/injection) were inoculated s.c. into the right flanks of nude mice (5-6 weeks old, female, Shanghai Slac Laboratory Animal Co. Ltd., Shanghai). When tumors were palpable, mice were randomly divided into two groups $(n=5)$. One group was orally administrated C96 $(100 \mathrm{mg} / \mathrm{kg}$ body weight, or $1 / 10$ oral $\mathrm{LD}_{50}$ ) dissolved in PBS containing $10 \%$ Tween 80 and DMSO daily for continued 16 days, another group was received the vehicle only. Tumor sizes were measured every other day with a caliper and calculated according to the formula $\left(\mathrm{V}=0.5236 a^{2} b\right.$, where $a$ is the smallest superficial diameter and $b$ is the largest superficial diameter) $[6,36]$. Body weight of each mouse was also monitored. At the end of the experiment, mice were sacrificed in a $\mathrm{CO}_{2}$ chamber containing dry ice. Tumor tissues were then excised, weighed, and snapfrozen in liquid nitrogen for further studies. To examine protein expression, tumor tissues were minced and ultrasonicated in the lysis buffer to extract proteins, which were further applied for immunoblotting analyses using specific antibodies against AKT, p-AKT, p-mTOR, mTOR, and GAPDH. This xenograft study was carried out in accordance with The Code of Ethics of the World Medical Association (Declaration of Helsinki) for experiments in animals and it was approved by the Animal Ethics and Welfare Review Board of Soochow University.

\section{ACKNOWLEDGEMENTS}

This project was supported by the Natural Science Foundation of China $(81071935,81272632$, $81101795,81301906,81320108023)$, the Natural Science Foundation of Jiangsu Province (BK2010218, BK2011268), by the National Basic Research Program of China (2011CB933501), and by the Priority Academic Program Development of Jiangsu Higher Education Institutions (PAPD). Dr. Xinliang Mao is a member of the Collaborative Innovation Center of Hematology, China.

\section{CONFLICT OF INTEREST}

There is no conflict of interest to declare for this study.

\section{REFERENCES}

1. Kyle RA and Rajkumar SV: Multiple myeloma. Blood. 2008; 111(6):2962-2972.

2. Kyle RA, Gertz MA, Witzig TE, Lust JA, Lacy MQ, Dispenzieri A, Fonseca R, Rajkumar SV, Offord JR and Larson DR. Review of 1027 patients with newly diagnosed multiple myeloma. Mayo Clin Proc. 2003; 78(1):21-33.

3. Cao B, Li J and Mao X. Dissecting bortezomib: development, application, adverse effects and future direction. Curr Pharm Des. 2013; 19(18):3190-3200.

4. Herndon TM, Deisseroth A, Kaminskas E, Kane RC, Koti KM, Rothmann MD, Habtemariam B, Bullock J, Bray JD and Hawes J. U.s. Food and drug administration approval: carfilzomib for the treatment of multiple myeloma. Clin Cancer Res. 2013; 19(17):4559-4563.

5. Li J, Cao B, Zhou S, Zhu J, Zhang Z, Hou T and Mao X. Cyproheptadine-induced myeloma cell apoptosis is associated with inhibition of the PI3K/AKT signaling. Eur J Haematol. 2013.

6. Mao X, Cao B, Wood TE, Hurren R, Tong J, Wang X, Wang W, Li J, Jin Y and Sun W. A small-molecule inhibitor of D-cyclin transactivation displays preclinical efficacy in myeloma and leukemia via phosphoinositide 3-kinase pathway. Blood. 2011; 117(6):1986-1997.

7. So L and Fruman DA. PI3K signalling in B- and T-lymphocytes: new developments and therapeutic advances. Biochem J. 2012; 442(3):465-481. 
8. Ikeda H, Hideshima T, Fulciniti M, Perrone G, Miura N, Yasui H, Okawa Y, Kiziltepe T, Santo L and Vallet S. $\mathrm{PI} 3 \mathrm{~K} / \mathrm{p} 110\{$ delta $\}$ is a novel therapeutic target in multiple myeloma. Blood. 2010; 116(9):1460-1468.

9. Glauer J, Pletz N, Schon M, Schneider P, Liu N, Ziegelbauer K, Emmert S, Wulf GG and Schon MP. A novel selective small-molecule PI3K inhibitor is effective against human multiple myeloma in vitro and in vivo. Blood Cancer J. 2013; :3:e141.

10. Chiron D, Maiga S, Surget S, Descamps G, GomezBougie P, Traore S, Robillard N, Moreau P, Le Gouill S and Bataille R. Autocrine insulin-like growth factor 1 and stem cell factor but not interleukin 6 support self-renewal of human myeloma cells. Blood Cancer J. 2013; :3:e120.

11. Zhang L, Yang J, Qian J, Li H, Romaguera JE, Kwak LW, Wang M and Yi Q. Role of the microenvironment in mantle cell lymphoma: IL-6 is an important survival factor for the tumor cells. Blood. 2012; 120(18):3783-3792.

12. Billottet C, Banerjee L, Vanhaesebroeck B and Khwaja A. Inhibition of class I phosphoinositide 3-kinase activity impairs proliferation and triggers apoptosis in acute promyelocytic leukemia without affecting atra-induced differentiation. Cancer Res. 2009; 69(3):1027-1036.

13. Gan F, Cao B, Wu D, Chen Z, Hou T and Mao X. Exploring old drugs for the treatment of hematological malignancies. Curr Med Chem. 2011; 18(10):1509-1514.

14. Frederick R, Mawson C, Kendall JD, Chaussade C, Rewcastle GW, Shepherd PR and Denny WA. Phosphoinositide-3-kinase (PI3K) inhibitors: identification of new scaffolds using virtual screening. Bioorg Med Chem Lett. 2009; 19(20):5842-5847.

15. Castillo JJ, Iyengar M, Kuritzky B and Bishop KD. Isotypespecific inhibition of the phosphatidylinositol-3-kinase pathway in hematologic malignancies. Onco Targets Ther. 2014; 7:333-342.

16. Walker EH, Perisic O, Ried C, Stephens L and Williams RL. Structural insights into phosphoinositide 3-kinase catalysis and signalling. Nature. 1999; 402(6759):313-320.

17. Knight ZA, Gonzalez B, Feldman ME, Zunder ER, Goldenberg DD, Williams O, Loewith R, Stokoe D, Balla A and Toth B. A pharmacological map of the PI3-K family defines a role for p110alpha in insulin signaling. Cell. 2006; 125(4):733-747.

18. Liu P, Cheng H, Roberts TM and Zhao JJ. Targeting the phosphoinositide 3-kinase pathway in cancer. Nat Rev Drug Discov. 2009; 8(8):627-644.

19. Petroulakis E, Mamane Y, Le B, acquer O, Shahbazian D and Sonenberg N. mTOR signaling: implications for cancer and anticancer therapy. Br J Cancer. 2006; 94(2): 195-199.

20. Han K, Xu X, Chen G, Zeng Y, Zhu J, Du X, Zhang Z, Cao B, Liu Z and Mao X. Identification of a promising PI3K inhibitor for the treatment of multiple myeloma through the structural optimization. J Hematol Oncol. 2014; 7(1):9.
21. Harvey RD and Lonial S. PI3 kinase/AKT pathway as a therapeutic target in multiple myeloma. Future Oncol. 2007; 3(6):639-647.

22. Li J, Zhu J, Cao B and Mao X. The mTOR Signaling Pathway is an Emerging Therapeutic Target in Multiple Myeloma. Curr Pharm Des. 2014; 20:125-35.

23. Lee G, Ramirez CN, Kim H, Zeltner N, Liu B, Radu C, Bhinder B, Kim YJ, Choi IY and Mukherjee-Clavin B. Large-scale screening using familial dysautonomia induced pluripotent stem cells identifies compounds that rescue IKBKAP expression. Nat Biotechnol. 2012; 30(12): 1244-1248.

24. Mao X, Stewart AK, Hurren R, Datti A, Zhu X, Zhu Y, Shi C, Lee K, Tiedemann R and Eberhard Y. A chemical biology screen identifies glucocorticoids that regulate c-maf expression by increasing its proteasomal degradation through up-regulation of ubiquitin. Blood. 2007; 110(12): 4047-4054.

25. Link W, Oyarzabal J, Serelde BG, Albarran MI, Rabal O, Cebria A, Alfonso P, Fominaya J, Renner $\mathrm{O}$ and Peregrina S. Chemical interrogation of FOXO3a nuclear translocation identifies potent and selective inhibitors of phosphoinositide 3-kinases. J Biol Chem. 2009; 284(41): 28392-28400.

26. Pinson JA, Schmidt-Kittler O, Zhu J, Jennings IG, Kinzler KW, Vogelstein B, Chalmers DK and Thompson PE. Thiazolidinedione-based PI3Kalpha inhibitors: an analysis of biochemical and virtual screening methods. ChemMed Chem. 2011; 6(3):514-522.

27. Park H, Lee S and Hong S. Discovery of MEK/PI3K dual inhibitor via structure-based virtual screening. Bioorg Med Chem Lett. 2012; 22(15):4946-4950.

28. Apsel B, Blair JA, Gonzalez B, Nazif TM, Feldman ME, Aizenstein B, Hoffman R, Williams RL, Shokat KM and Knight ZA. Targeted polypharmacology: discovery of dual inhibitors of tyrosine and phosphoinositide kinases. Nat Chem Biol. 2008; 4(11):691-699.

29. Hennessy BT, Smith DL, Ram PT, Lu Y and Mills GB. Exploiting the PI3K/AKT pathway for cancer drug discovery. Nat Rev Drug Discov. 2005; 4(12):988-1004.

30. Zhu J, Pan P, Li Y, Wang M, Li D, Cao B, Mao X and Hou T. Theoretical studies on beta and delta isoformspecific binding mechanisms of phosphoinositide 3-kinase inhibitors. Mol Biosyst. 2014; 10(3):454-66.

31. Venable JD, Ameriks MK, Blevitt JM, Thurmond RL and Fung-Leung WP. Phosphoinositide 3-kinase gamma (PI3Kgamma) inhibitors for the treatment of inflammation and autoimmune disease. Recent Pat Inflamm Allergy Drug Discov. 2014; 4(1):1-15.

32. Fukushima T, Nakamura Y, Yamanaka D, Shibano T, Chida K, Minami S, Asano T, Hakuno F and Takahashi S. Phosphatidylinositol 3-kinase (PI3K) activity bound to insulin-like growth factor-I (IGF-I) receptor, which is continuously sustained by IGF-I stimulation, is required 
for IGF-I-induced cell proliferation. J Biol Chem. 2012; 287(35):29713-29721.

33. Pennarun B, Kleibeuker JH, Oenema T, Stegehuis JH, de Vries EG and de Jong S. Inhibition of IGF-1R-dependent PI3K activation sensitizes colon cancer cells specifically to DR5-mediated apoptosis but not to rhTRAIL. Cell Oncol (Dordr). 2011; 34(3):245-259.

34. Georgakis GV, Li Y, Rassidakis GZ, Medeiros LJ, Mills GB and Younes A. Inhibition of the phosphatidylinositol-3 kinase/Akt promotes G1 cell cycle arrest and apoptosis in Hodgkin lymphoma. Br J Haematol. 2006; 132(4): 503-511.

35. Ohwada J, Ebiike H, Kawada H, Tsukazaki M, Nakamura M, Miyazaki T, Morikami K, Yoshinari K,
Yoshida $\mathrm{M}$ and Kondoh O. Discovery and biological activity of a novel class I PI3K inhibitor, CH5132799. Bioorg Med Chem Lett. 2011; 21(6):1767-1772.

36. Ling C, Chen G, Chen G, Zhang Z, Cao B, Han K, Yin J, Chu A, Zhao Y and Mao X. A deuterated analog of dasatinib disrupts cell cycle progression and displays antinon-small cell lung cancer activity in vitro and in vivo. Int J Cancer. 2012; 131(10):2411-2419.

37. Tai YT, Podar K, Mitsiades N, Lin B, Mitsiades C, Gupta D, Akiyama M, Catley L, Hideshima $\mathrm{T}$ and Munshi NC. CD40 induces human multiple myeloma cell migration via phosphatidylinositol 3-kinase/AKT/NF-kappa B signaling. Blood. 2003; 101(7):2762-2769. 